\title{
Bone marrow fibrosis in childhood acute lymphoblastic leukaemia
}

\author{
J P WALLIS, M M REID \\ From the Department of Haematology, Royal Victoria Infirmary, Newcastle upon Tyne
}

SUMMARY Bone marrow trephine biopsy specimens were obtained at diagnosis from 63 of 76 consecutively presenting children with acute lymphoblastic leukaemia (ALL). The association between marrow fibrosis and presenting features, including immunophenotype, was analysed. Reticulin was increased in 45 of 56 cases in which blasts expressed B lineage markers, but in only one of seven with T-ALL. A weak association was also found between marrow fibrosis and splenomegaly in those with common ALL. Marrow fibrosis is apparently associated with some examples of ALL of B cell lineage, but precisely which subtypes and whether the phenomenon is clinically important remain to be determined.

Secondary bone marrow fibrosis occurs in a variety of conditions including both myeloid and lymphoblastic leukaemia.' It is also a particular feature of some B cell neoplasms such as hairy cell leukaemia and Waldenstrom's macroglobulinaemia.

This study was undertaken to assess what clinical importance the presence or absence of marrow fibrosis might have in acute lymphoblastic leukaemia (ALL) in children. A previous study by Hann et al in 1978 concluded that there was no significant association with age, sex, white cell count or organomegaly. ${ }^{2}$ There was a tendency to better survival in those with increased fibrosis which did not quite reach significance. Treatment is now more aggressive and immunophenotyping has improved considerably. Since late 1984 it has been our policy to perform routinely bone marrow biopsy in children with ALL at diagnosis. We made a retrospective study of marrow fibrosis in these children, paying particular attention to the immunophenotype of the leukaemia.

\section{Patients and methods}

Seventy six children of less than 15 years of age with ALL presented or were referred to the Royal Victoria Infirmary in Newcastle upon Tyne between August 1984 and March 1989. Of these, 63 had trephine biopsies of the anterior iliac crest performed under general anaesthetic using Jamshidi paediatric trephine biopsy needles. Thirteen cases did not have biopsy specimens available for study: two had diagnostic marrow aspirates only which were performed at other hospitals; three did not have a general anaesthetic because of poor clinical condition; three with very high white cell counts did not have a bone marrow examination (two T-cell, one CALL); in four cases a haematologist skilled in performing trephine biopsies in children was not available and biopsy was not attempted; and in one case the material was mislaid. These cases did not form a particular clinical or diagnostic group (three T-cell, eight CALL, two other). Sixty children were treated with the MRC UKALL $X$ protocol and three with the UKALL VIII protocol.

Biopsy specimens were fixed in formol saline, decalcified in Gooding's and Stuart's solution for eight hours, and paraffin wax embedded sections were stained by Foot's silver reticulin method. Reticulin was assessed independently by two observers and graded as not increased $(0)$, slightly increased $(+)$, moderately increased $(++)$, or greatly increased $(+++)$. Assessment was made with no knowledge of the patient's identity or other details and differed between the two observers in 24 of the 63 cases by a single grade. In these cases the trephine biopsy specimen was re-examined jointly and agreement reached.

Immunophenotyping was performed by standard indirect immunofluorescence labelling of bone marrow mononuclear cell suspensions. CD10 (CALLA), CD19 (B4), CD20 (B1), and CD2 (T11) were routinely assessed in most patients. CD1 (T6), CD4 (T4), CD8 (T8), CD7 (Leu 9), CD13 (MY 7), CD14 (MY 4), CD33 (MY 9), surface membrane immunoglobulin, and glycoprotein IIb/IIIa were also tested in several patients. Terminal deoxynucleotidyl transferase (Tdt) was assessed by indirect immunofluorescence using a polyclonal rabbit anti-Tdt antibody on a fresh marrow slide or marrow cytospin in all cases. Cytoplasmic $\mu$ has only been tested for more recently and results are not included in the analyses. Immunophenotypes were defined as follows:

1 Common $A L L$ (CALL): Tdt positive, CD10 positive, $\mathrm{T}$ cell markers negative (in all but four cases $>30 \%$ of blasts were CD19 or CD20 positive).

2 T cell $A L L(T-A L L): \mathrm{CD} 2, \mathrm{CD} 1$, or $\mathrm{CD} 7$ positive, 
Table Association between immunophenotype and reticulin in childhood ALL

\begin{tabular}{lccccc}
\hline Reticulin & 0 & + & ++ & +++ & Total \\
CALL & 8 & 18 & 15 & 6 & 47 \\
TALL & 6 & 1 & 0 & 0 & 7 \\
Other* & 3 & 3 & 1 & 2 & 9 \\
\hline
\end{tabular}

*Comprise three Tdt negative, $\mathrm{CD} 10+$; one Tdt +, CD19 +, CD10 negative; two coexpression of $B$ and T markers; two Burkitt's with 14 $q+$ marker; and one non-marking leukaemia with $t(4: 11)$.

CD19 and CD20 negative. Tdt positive or negative.

3 Other ALL: (table).

Details of the peripheral blood count were taken from the presenting blood sample. Splenomegaly and lymphadenopathy were as recorded at initial presentation.

Cytogenetic analyses were performed on unstimulated bone marrow cultures and metaphase cells stained by standard G-banding techniques.

Statistical analysis was performed using Fisher's exact probability test.

\section{Results}

Sixty three trephine biopsy specimens were studied. The table shows the assessment of reticulin according to immunophenotype.

The groups were divided into T-ALL, and those with B lineage markers (CALL and others), and into no fibrosis $(0)$ and fibrosis $(+,++,+++)$ for statistical analysis. The association between leukaemia with B lineage markers and presence of fibrosis was significant $(2 p<0.005)$. There was no trend or significant difference in presenting haemoglobin (mean $7 \mathrm{~g} / \mathrm{dl}$ ), mean corpuscular volume (mean 83f), or platelet count when analysed according to immunophenotype or degree of fibrosis. White cell count was higher for the group without fibrosis but this trend disappeared when analysed for CALL alone. Splenomegaly (35 of 63) showed no significant association with fibrosis, though when analysed for CALL alone, splenomegaly was less common in those without fibrosis (one of eight) than in those with fibrosis (23 of 39). This difference was just significant $(2 p<0.05)$.

Chromosomes were successfully studied in 44 of 63 cases. No association between particular abnormalities or groups of abnormalities with degree of fibrosis could be discerned. Seven of the 63 children have relapsed since the presenting marrow biopsy was obtained (median relapse free follow up 21 months) and three children died during or failed induction. These show no significant association with degree of fibrosis. Of 24 cases with moderate or pronounced increase in reticulin staining 17 had repeat trephine biopsies performed 30 days after starting treatment and, in all but two, reticulin had returned to normal.

\section{Discussion}

Marrow fibrosis was found in 46 of 63 children with
ALL, an incidence similar to that found by Kundel $e t$ $a l^{1}$ and by Hann et al in $1978 .{ }^{2}$ Since their study the use of monoclonal antibodies has permitted more precise immunophenotyping of acute leukaemia and advances in treatment have considerably improved the prognosis of childhood ALL.

The principal finding of our study was the strong association between the expression of $B$ lineage markers and presence of marrow fibrosis. More careful inspection of the immunophenotypes shows that three cases with marrow fibrosis showed expression of $\mathrm{T}$ cell markers. One with a moderate and one with a pronounced increase in reticulin both showed coexpression of $\mathrm{T}$ and $\mathrm{B}$ cell markers including CD10 and were classified as "other". One with a slight increase in reticulin, expressing Tdt $95 \%, \mathrm{CD} 1040 \%$, CD1 92\%, CD7 40\%, CD2 5\%, CD19 and CD20 $<1 \%$ was classified as T-ALL. No other case of T-ALL expressed CD10. One case of null cell leukaemia with $\mathrm{t}(4: 11)$ and a slight increase in reticulin later relapsed expressing CD19. All other cases with fibrosis were CALL or expressed only B cell markers at diagnosis. There was no association between "maturity" of B cell markers and degree of fibrosis in either the CALL or "other" groups. The proportion of T-ALL among the total was similar to that previously found in the Northern region ${ }^{3}$ and elsewhere in the United Kingdom. ${ }^{4}$

Apart from a weak association between splenomegaly and fibrosis in CALL, no independent correlation was found between degree of fibrosis and other? presenting features. In particular, the association between absence of fibrosis and high presenting white cell count seemed to reflect the distribution of T-ALL.

Improvements in the treatment of childhood ALL have resulted in fewer relapses such that considerably longer follow up of larger series of patients is required before any association between prognosis and fibrosis might be determined. Despite the small size of this group, there does seem to be a biological difference in the ability of distinct immunologically defined subgroups of ALL to stimulate a fibrotic reaction in the marrow.

\section{References}

1 Kundel DW, Brecher G, Bodey GP, Brittin GM. Reticulin fibrosis and bone infarction in acute leukemia. Implications for prognosis. Blood 1964;23:526-44.

2 Hann IM, Evans DIK, Marsden HB, Morris-Jones P, Palmer MK Bone marrow fibrosis in acute lymphoblastic leukaemia of childhood. J Clin Pathol 1978;31:313-5.

3 Reid MM, Craft AW, Walker W. Poor prognosis of T-cell leukaemia in childhood. Lancet 1977;ii:1074.

4 Chessells JM, Hardisty RM, Rapson NT, Greaves MF. Acute lymphoblastic leukaemia in childhood: Classification and Prognosis. Lancet 1977;ii:1307-9.

Requests for reprints to: Dr J P Wallis, Department of Haematology, Royal Victoria Infirmary, Newcastle upon Tyne NE1 4LP, England. 